\title{
The existence of fixed point theorems for partial $q$-set-valued quasi-contractions in $b$-metric spaces and related results
}

\author{
Poom Kumam ${ }^{1}$ and Wutiphol Sintunavarat ${ }^{2 *}$
}

\section{"Correspondence:}

wutiphol@mathstat.sci.tu.ac.th; poom_teun@hotmail.com

${ }^{2}$ Department of Mathematics and

Statistics, Faculty of Science and Technology, Thammasat University

Rangsit Center, Pathumthani, 12121, Thailand

Full list of author information is available at the end of the article

\begin{abstract}
In this paper, we present a new type of set-valued mappings called partial $q$-set-valued quasi-contraction mappings and give results as regards fixed points for such mappings in $b$-metric spaces. By providing some examples, we show that our results are real generalizations of the main results of Aydi et al. (Fixed Point Theory Appl. 2012:88, 2012) and many results in the literature. We also consider fixed point results for single-valued mapping, fixed point results for set-valued mapping in $b$-metric space endowed with an arbitrary binary relation, and fixed point results in a $b$-metric space endowed with a graph. By using our result, we establish the existence of solution for the following an integral equations: $x(c)=\phi(c)+\int_{a}^{b} K(c, r, x(r)) d r$, where $b>a \geq 0, x \in C[a, b]$ (the set of continuous real functions defined on $[a, b] \subseteq \mathbb{R}$ ), $\phi:[a, b] \rightarrow \mathbb{R}$, and $K:[a, b] \times[a, b] \times \mathbb{R} \rightarrow \mathbb{R}$ are given mappings.
\end{abstract}

MSC: $47 \mathrm{H} 10 ; 54 \mathrm{H} 25$

Keywords: $\alpha$-admissible mappings; binary relations; fixed points; $b$-metric spaces; q-set-valued $\alpha$-quasi-contraction mappings

\section{Introduction}

The Banach contraction principle is a very popular tool of mathematics in solving many problems in several branches of mathematics since it can be observed easily and comfortably. In 1993, Czerwik [1] introduced the concept of $b$-metric spaces and also presented the fixed point theorem for contraction mappings in $b$-metric spaces, that is, we have a generalization of the Banach contraction principle in metric spaces. Afterward, many mathematicians studied fixed point theorems for single-valued and set-valued mappings in $b$-metric spaces (see [2-7] and references therein).

In 2012, Aydi et al. [8] extended the concept of $q$-set-valued quasi-contraction mappings in metric spaces due to Amini-Harandi [9] to $b$-metric spaces. They also established the fixed point results for $q$-set-valued quasi-contraction mappings in $b$-metric spaces. Recently, Sintunavarat et al. [10] introduced some set-valued mappings called $q$-set-valued $\alpha$-quasi-contraction mappings and obtained fixed point results for such mappings in $b$ metric spaces which are generalization of the results of Aydi et al. [8], Amini-Harandi [9] and many works in the literature.

Inspired and motivated by several results in the literature, we introduce the class of partial $q$-set-valued quasi-contraction mappings which is the wider class of many classes in

\section{空 Springer}

(02014 Kumam and Sintunavarat; licensee Springer. This is an Open Access article distributed under the terms of the Creative Commons Attribution License (http://creativecommons.org/licenses/by/2.0), which permits unrestricted use, distribution, and reproduction in any medium, provided the original work is properly cited. 
this field. As regards this class, we study and obtain fixed point results in $b$-metric spaces. These results extend, unify and generalize several well-known comparable results in the existing literature. As an application of our results, we prove the fixed point theorems for a single-valued mapping and give an example to show the generality of our result. We also study the fixed point results in a $b$-metric space endowed with an arbitrary binary relation and endowed with a graph. As applications, we apply our result to the proof of the existence of a solution for the following an integral equation:

$$
x(c)=\phi(c)+\int_{a}^{b} K(c, r, x(r)) d r
$$

where $b>a \geq 0, x \in C[a, b]$ (the set of continuous real functions defined on $[a, b] \subseteq \mathbb{R}$ ), $\phi:[a, b] \rightarrow \mathbb{R}$, and $K:[a, b] \times[a, b] \times \mathbb{R} \rightarrow \mathbb{R}$ are given mappings.

\section{Preliminaries}

In this section, we give some notations and basic knowledge in nonlinear analysis and $b$ metric spaces. Throughout this paper, $\mathbb{R}, \mathbb{R}_{+}$, and $\mathbb{N}$ denote the set of real numbers, the set of nonnegative real numbers, and the set of positive integers, respectively.

Definition 2.1 ([1]) Let $X$ be a nonempty set and $s \geq 1$ be a given real number. A functional $d: X \times X \rightarrow \mathbb{R}_{+}$is called a $b$-metric if, for all $x, y, z \in X$, the following conditions are satisfied:

$\left(\mathrm{B}_{1}\right) d(x, y)=0$ if and only if $x=y$;

$\left(\mathrm{B}_{2}\right) d(x, y)=d(y, x)$;

$\left(\mathrm{B}_{3}\right) d(x, z) \leq s[d(x, y)+d(y, z)]$.

A pair $(X, d)$ is called a $b$-metric space with coefficient $s$.

Remark 2.2 The result is obtained that any metric space is a $b$-metric space with $s=1$. Thus the class of $b$-metric spaces is larger than the class of metric spaces.

Some examples of $b$-metric spaces are given by Berinde [11], Czerwik [6], Heinonen [12]. Some well-known examples of a $b$-metric which show that the $b$-metric space is a real generalization of metric space are the following.

Example 2.3 The set of real numbers together with the functional $d: \mathbb{R} \times \mathbb{R} \rightarrow \mathbb{R}_{+}$,

$$
d(x, y):=|x-y|^{2}
$$

for all $x, y \in \mathbb{R}$, is a $b$-metric space with coefficient $s=2$. However, we find that $d$ is not a metric on $X$ since the ordinary triangle inequality is not satisfied. Indeed,

$$
d(2,4)>d(2,3)+d(3,4) .
$$

Example 2.4 Let $(X, d)$ be a metric space and a functional $\rho: \mathbb{R} \times \mathbb{R} \rightarrow \mathbb{R}_{+}$defined by $\rho(x, y)=(d(x, y))^{p}$, where $p>1$ is a fixed real number. We show that $\rho$ is a $b$-metric with 
$s=2^{p-1}$. It is easy to see that conditions $\left(\mathrm{B}_{1}\right)$ and $\left(\mathrm{B}_{2}\right)$ are satisfied. If $1<p<\infty$, then the convexity of the function $f(x)=x^{p}(x>0)$ implies the following inequality:

$$
\left(\frac{a+b}{2}\right)^{p} \leq \frac{1}{2}\left(a^{p}+b^{p}\right)
$$

that is,

$$
(a+b)^{p} \leq 2^{p-1}\left(a^{p}+b^{p}\right)
$$

holds. Therefore, for each $x, y, z \in X$, we get

$$
\begin{aligned}
\rho(x, y) & =(d(x, y))^{p} \\
& \leq(d(x, z)+d(z, y))^{p} \\
& \leq 2^{p-1}\left((d(x, z))^{p}+(d(z, y))^{p}\right) \\
& =2^{p-1}(\rho(x, z)+\rho(z, y)) .
\end{aligned}
$$

Consequently, condition $\left(\mathrm{B}_{3}\right)$ is also satisfied and thus $\rho$ is a $b$-metric on $X$.

Example 2.5 The set $l_{p}(\mathbb{R})$ with $0<p<1$, where

$$
l_{p}(\mathbb{R}):=\left\{\left.\left\{x_{n}\right\} \subseteq \mathbb{R}\left|\sum_{n=1}^{\infty}\right| x_{n}\right|^{p}<\infty\right\}
$$

together with the functional $d: l_{p}(\mathbb{R}) \times l_{p}(\mathbb{R}) \rightarrow \mathbb{R}_{+}$,

$$
d(x, y):=\left(\sum_{n=1}^{\infty}\left|x_{n}-y_{n}\right|^{p}\right)^{\frac{1}{p}}
$$

for each $x=\left\{x_{n}\right\}, y=\left\{y_{n}\right\} \in l_{p}(\mathbb{R})$, is a $b$-metric space with coefficient $s=2^{\frac{1}{p}}>1$. We see that the above result also holds for the general case $l_{p}(X)$ with $0<p<1$, where $X$ is a Banach space.

Example 2.6 Let $p$ be a given real number in the interval $(0,1)$. The space $L_{p}[0,1]$ of all real functions $x(t), t \in[0,1]$ such that $\int_{0}^{1}|x(t)|^{p} d t<1$, together with the functional $d$ : $L_{p}[0,1] \times L_{p}[0,1] \rightarrow \mathbb{R}_{+}$,

$$
d(x, y):=\left(\int_{0}^{1}|x(t)-y(t)|^{p} d t\right)^{1 / p}, \quad \text { for each } x, y \in L_{p}[0,1]
$$

is a $b$-metric space with constant $s=2^{\frac{1}{p}}$.

Example 2.7 Let $X=\{0,1,2\}$ and a functional $d: X \times X \rightarrow \mathbb{R}_{+}$be defined by

$$
\begin{aligned}
& d(0,0)=d(1,1)=d(2,2)=0 \\
& d(0,1)=d(1,0)=d(1,2)=d(2,1)=1
\end{aligned}
$$


and

$$
d(2,0)=d(0,2)=m \text {, }
$$

where $m$ is given real number such that $m \geq 2$. It easy to see that

$$
d(x, y) \leq \frac{m}{2}[d(x, z)+d(z, y)]
$$

for all $x, y, z \in X$. Therefore, $(X, d)$ is a $b$-metric space with coefficient $s=m / 2$. We find that the ordinary triangle inequality does not hold if $m>2$ and then $(X, d)$ is not a metric space.

Next, we give the concepts of convergence, compactness, closedness, and completeness in a $b$-metric space.

Definition 2.8 ([4]) Let $(X, d)$ be a $b$-metric space. The sequence $\left\{x_{n}\right\}$ in $X$ is called:

(1) convergent if and only if there exists $x \in X$ such that $d\left(x_{n}, x\right) \rightarrow 0$ as $n \rightarrow \infty$. In this case, we write $\lim _{n \rightarrow \infty} x_{n}=x$.

(2) Cauchy if and only if $d\left(x_{n}, x_{m}\right) \rightarrow 0$ as $m, n \rightarrow \infty$.

Remark 2.9 In a $b$-metric space $(X, d)$ the following assertions hold:

(1) a convergent sequence has a unique limit;

(2) each convergent sequence is Cauchy;

(3) in general a functional $b$-metric $d: X \times X \rightarrow \mathbb{R}_{+}$for coefficient $s>1$ is not jointly continuous in all its variables.

The following example is an example of a $b$-metric which is not continuous.

Example 2.10 (see [13]) Let $X=\mathbb{N} \cup\{\infty\}$ and a functional $d: X \times X \rightarrow \mathbb{R}_{+}$be defined by

$$
d(x, y)= \begin{cases}0, & x=y, \\ \frac{1}{y}, & x=\infty \text { and } y \neq \infty, \\ \frac{1}{x}, & x \neq \infty \text { and } y=\infty, \\ \left|\frac{1}{x}-\frac{1}{y}\right|, & x \text { and } y \text { are even, } \\ 5, & x \text { and } y \text { are odd and } x \neq y, \\ 2, & \text { otherwise. }\end{cases}
$$

It is easy to see that conditions $\left(\mathrm{B}_{1}\right)$ and $\left(\mathrm{B}_{2}\right)$ are satisfied. Also, for each $x, y, z \in X$, we have

$$
d(x, z) \leq 3[d(x, y)+d(y, z)]
$$

Therefore, $(X, d)$ is a $b$-metric space on $X$ with coefficient $s=3$.

Next, we show that $d$ is not continuous. Let $x_{n}=2 n$ for each $n \in \mathbb{N}$. It is easy to see that

$$
d\left(x_{n}, \infty\right)=d(2 n, \infty)=\frac{1}{2 n} \rightarrow 0, \quad \text { as } n \rightarrow \infty,
$$

that is, $x_{n} \rightarrow \infty$, but $d\left(x_{n}, 1\right)=2 \nrightarrow d(\infty, 1)$ as $n \rightarrow \infty$. Therefore, $d$ is not continuous. 
Definition 2.11 The $b$-metric space $(X, d)$ is complete if every Cauchy sequence in $X$ converges.

Definition 2.12 ([4]) Let $Y$ be a nonempty subset of a $b$-metric space $X$. The closure $\bar{Y}$ of $Y$ is the set of limits of all convergent sequences of points in $Y$, i.e.,

$$
\bar{Y}:=\left\{x \in X: \text { there exists a sequence }\left\{x_{n}\right\} \text { in } Y \text { such that } \lim _{n \rightarrow \infty} x_{n}=x\right\} .
$$

Definition 2.13 ([4]) Let $(X, d)$ be a $b$-metric space. A subset $Y \subseteq X$ is called:

(1) closed if and only if for each sequence $\left\{x_{n}\right\}$ in $Y$ which converges to an element $x$, we have $x \in Y$ (i.e. $Y=\bar{Y}$ );

(2) compact if and only if for every sequence of element in $Y$ there exists a subsequence that converges to an element in $Y$;

(3) bounded if and only if $\delta(Y):=\sup \{d(a, b) \mid a, b \in Y\}<\infty$.

Throughout this paper, we use the following notations of collection of subsets of a $b$ metric space $(X, d)$ :

$$
\begin{aligned}
& \mathcal{P}(X):=\{Y \mid Y \subseteq X\} ; \\
& P(X):=\{Y \in \mathcal{P}(X) \mid Y \neq \emptyset\} ; \\
& P_{b}(X):=\{Y \in P(X) \mid Y \text { is bounded }\} ; \\
& P_{c p}(X):=\{Y \in P(X) \mid Y \text { is compact }\} ; \\
& P_{c l}(X):=\{Y \in P(X) \mid Y \text { is closed }\} ; \\
& P_{b, c l}(X):=P_{b}(X) \cap P_{c l}(X) .
\end{aligned}
$$

Next, we give the concept of generalized functionals on a $b$-metric space $(X, d)$.

Definition 2.14 Let $(X, d)$ be a $b$-metric space.

(1) The functional $D: \mathcal{P}(X) \times \mathcal{P}(X) \rightarrow \mathbb{R} \cup\{+\infty\}$ is said to be a gap functional if and only if it is defined by

$$
D(A, B)= \begin{cases}\inf \{d(a, b) \mid a \in A, b \in B\}, & A \neq \emptyset \neq B \\ 0, & A=\emptyset=B \\ +\infty, & \text { otherwise }\end{cases}
$$

In particular, if $x_{0} \in X$ then $d\left(x_{0}, B\right):=D\left(\left\{x_{0}\right\}, B\right)$.

(2) The functional $\rho: \mathcal{P}(X) \times \mathcal{P}(X) \rightarrow \mathbb{R} \cup\{+\infty\}$ is said to be an excess generalized functional if and only if it is defined by

$$
\rho(A, B)= \begin{cases}\sup \{d(a, B) \mid a \in A\}, & A \neq \emptyset \neq B, \\ 0, & A=\emptyset, \\ +\infty, & \text { otherwise }\end{cases}
$$


(3) The functional $H: \mathcal{P}(X) \times \mathcal{P}(X) \rightarrow \mathbb{R} \cup\{+\infty\}$ is said to be a Pompeiu-Hausdorff generalized functional if and only if it is defined by

$$
H(A, B)= \begin{cases}\max \{\rho(A, B), \rho(B, A)\}, & A \neq \emptyset \neq B \\ 0, & A=\emptyset, \\ +\infty, & \text { otherwise }\end{cases}
$$

Remark 2.15 For $b$-metric space $(X, d)$, the following assertions hold:

(1) $\left(P_{c p}(X), H\right)$ is a complete $b$-metric space provided $(X, d)$ is a complete $b$-metric space;

(2) for each $A, B \in P(X)$ and $x \in A$, we have

$$
d(x, B) \leq \rho(A, B) \leq H(A, B)
$$

(3) for $x \in X$ and $B \in P(X)$, we get

$$
d(x, B) \leq d(x, b)
$$

for all $b \in B$.

The following lemmas are useful for the proofs in the main result.

Lemma $2.16([6])$ Let $(X, d)$ be a b-metric space. Then

$$
d(x, A) \leq s[d(x, B)+H(B, A)]
$$

for all $x \in X$ and $A, B \in P(X)$. In particular, we have

$$
d(x, A) \leq s[d(x, y)+d(y, A)]
$$

for all $x, y \in X$ and $A \in P(X)$.

Lemma 2.17 ([6]) Let $(X, d)$ be a b-metric space and $A, B \in P_{b, c l}(X)$. Then for each $\epsilon>0$ and, for all $b \in B$, there exists $a \in A$ such that $d(a, b) \leq H(A, B)+\epsilon$.

Lemma $2.18([6])$ Let $(X, d)$ be a b-metric space. For $A \in P_{b, c l}(X)$ and $x \in X$, we have

$$
d(x, A)=0 \quad \Longrightarrow \quad x \in A \text {. }
$$

Lemma 2.19 ([14]) Let $(X, d)$ be a b-metric space with coefficient $s \geq 1$ and $\left\{x_{n}\right\}$ be a sequence in $X$ such that

$$
d\left(x_{n+1}, x_{n+2}\right) \leq \gamma d\left(x_{n}, x_{n+1}\right)
$$

for all $n \in \mathbb{N}$, where $0 \leq \gamma<1$. Then $\left\{x_{n}\right\}$ is a Cauchy sequence in $X$ provided that $s \gamma<1$.

In 2012, Samet et al. [15] introduced the concepts of $\alpha$-admissible mapping as follows. 
Definition 2.20 ([15]) Let $X$ be a nonempty set, $t: X \rightarrow X$ and $\alpha: X \times X \rightarrow[0, \infty)$. We say that $t$ is $\alpha$-admissible if

$$
\text { for } x, y \in X \text { for which } \alpha(x, y) \geq 1 \quad \Longrightarrow \quad \alpha(t x, t y) \geq 1 \text {. }
$$

They proved the fixed point results for single-valued mapping as regards this concept and also showed that these results can be utilized to derive fixed point theorems in partially ordered spaces. As an application, they obtain the existence of solutions for ordinary differential equations.

Afterward, Asl et al. [16] and Mohammadi et al. [17] introduced the concept of $\alpha_{*^{-}}$ admissibility and $\alpha$-admissibility for set-valued mappings as follows.

Definition 2.21 ([16, 17]) Let $X$ be a nonempty set, $T: X \rightarrow 2^{X}$, where $2^{X}$ is a collection of nonempty subsets of $X$ and $\alpha: X \times X \rightarrow[0, \infty)$. We say that

(1) $T$ is $\alpha_{*}$-admissible if

$$
\text { for } x, y \in X \text { for which } \alpha(x, y) \geq 1 \quad \Longrightarrow \quad \alpha_{*}(T x, T y) \geq 1 \text {, }
$$

where $\alpha_{*}(T x, T y):=\inf \{\alpha(a, b) \mid a \in T x, b \in T y\}$.

(2) $T$ is $\alpha$-admissible if for each $x \in X$ and $y \in T x$ with $\alpha(x, y) \geq 1$, we have $\alpha(y, z) \geq 1$, for all $z \in T y$.

Remark 2.22 If $T$ is $\alpha_{*}$-admissible, then $T$ is also $\alpha$-admissible mapping.

In recent investigations, the fixed point results for single-valued and set-valued mappings via the concepts of being $\alpha$-admissible and $\alpha_{*}$-admissible occupies a prominent place in many aspects (see [18-25] and references therein).

\section{Fixed point theorems for partial $q$-set-valued quasi-contraction mappings}

In this section, we introduce the partial $q$-set-valued quasi-contraction mapping and obtain the theorem of the existence of a fixed point for such a mapping in $b$-metric spaces.

Throughout this paper, for the nonempty set $X$ and the given mapping $\alpha: X \times X \rightarrow$ $[0, \infty)$, we use the following notation:

$$
\bigwedge_{\alpha}:=\{(x, y) \in X \times X: \alpha(x, y) \geq 1\}
$$

Definition 3.1 Let $(X, d)$ be a $b$-metric space and $\alpha: X \times X \rightarrow[0, \infty)$ be a given mapping. The set-valued mapping $T: X \rightarrow P_{b, c l}(X)$ is said to be a partial q-set-valued quasicontraction if, for all $(x, y) \in X \times X$,

$$
\begin{aligned}
& (x, y) \in \bigwedge_{\alpha} \\
& \quad \Longrightarrow \quad H(T x, T y) \leq q \max \{d(x, y), d(x, T x), d(y, T y), d(x, T y), d(y, T x)\}
\end{aligned}
$$

where $0 \leq q<1$.

Next, we give the main result in this paper. 
Theorem 3.2 Let $(X, d)$ be a complete $b$-metric space with coefficient $s \geq 1, \alpha: X \times$ $X \rightarrow[0, \infty)$ be a given mapping and $T: X \rightarrow P_{b, c l}(X)$ be a partial q-set-valued quasicontraction. Suppose that the following conditions hold:

(i) $T$ is $\alpha$-admissible;

(ii) there exist $x_{0} \in X$ and $x_{1} \in T x_{0}$ such that $\left(x_{0}, x_{1}\right) \in \bigwedge_{\alpha}$;

(iii) if $\left\{x_{n}\right\}$ is a sequence in $X$ such that $\left(x_{n}, x_{n+1}\right) \in \bigwedge_{\alpha}$, for all $n \in \mathbb{N}$, and $x_{n} \rightarrow x$ as $n \rightarrow \infty$, for some $x \in X$, then $\left(x_{n}, x\right) \in \bigwedge_{\alpha}$.

If $q<\frac{1}{s^{2}+s}$, then $T$ has a fixed point in $X$, that is, there exists $u \in X$ such that $u \in T u$.

Proof For $x, y \in X$, we obtain

$$
\max \{d(x, y), d(x, T x), d(y, T y), d(x, T y), d(y, T x)\}=0
$$

if and only if $x=y$ is a fixed point of $T$. Therefore, we suppose that

$$
\max \{d(x, y), d(x, T x), d(y, T y), d(x, T y), d(y, T x)\}>0,
$$

for all $x, y \in X$.

Now, we will set

$$
\varepsilon:=\frac{1}{2}\left(\frac{1}{s^{2}+s}-q\right) \text { and } \beta:=q+\varepsilon=\frac{1}{2}\left(\frac{1}{s^{2}+s}+q\right) .
$$

It follows from $q<\frac{1}{s^{2}+s}$ that $\varepsilon>0$ and $0<\beta<\frac{1}{s^{2}+s}$.

Starting from $x_{0}$ and $x_{1} \in T x_{0}$ in (ii), by Lemma 2.17, there exists $x_{2} \in T x_{1}$ such that

$$
\begin{aligned}
d\left(x_{1}, x_{2}\right) \leq & H\left(T x_{0}, T x_{1}\right) \\
& +\varepsilon \max \left\{d\left(x_{0}, x_{1}\right), d\left(x_{0}, T x_{0}\right), d\left(x_{1}, T x_{1}\right), d\left(x_{0}, T x_{1}\right), d\left(x_{1}, T x_{0}\right)\right\} .
\end{aligned}
$$

It follows from $\left(x_{0}, x_{1}\right) \in \bigwedge_{\alpha}$ that

$$
H\left(T x_{0}, T x_{1}\right) \leq q \max \left\{d\left(x_{0}, x_{1}\right), d\left(x_{0}, T x_{0}\right), d\left(x_{1}, T x_{1}\right), d\left(x_{0}, T x_{1}\right), d\left(x_{1}, T x_{0}\right)\right\} .
$$

From (3.2) and (3.3), we get

$$
\begin{aligned}
d\left(x_{1}, x_{2}\right) & \leq(q+\varepsilon) \max \left\{d\left(x_{0}, x_{1}\right), d\left(x_{0}, T x_{0}\right), d\left(x_{1}, T x_{1}\right), d\left(x_{0}, T x_{1}\right), d\left(x_{1}, T x_{0}\right)\right\} \\
& =\beta \max \left\{d\left(x_{0}, x_{1}\right), d\left(x_{0}, T x_{0}\right), d\left(x_{1}, T x_{1}\right), d\left(x_{0}, T x_{1}\right), d\left(x_{1}, T x_{0}\right)\right\} .
\end{aligned}
$$

Since $T$ is $\alpha$-admissible, $x_{0} \in X$, and $x_{1} \in T x_{0}$ such that $\alpha\left(x_{0}, x_{1}\right) \geq 1$, we get $\alpha\left(x_{1}, x_{2}\right) \geq 1$ and so $\left(x_{1}, x_{2}\right) \in \bigwedge_{\alpha}$. Using Lemma 2.17, there exists $x_{3} \in T x_{2}$ such that

$$
\begin{aligned}
d\left(x_{2}, x_{3}\right) \leq & H\left(T x_{1}, T x_{2}\right) \\
& +\varepsilon \max \left\{d\left(x_{1}, x_{2}\right), d\left(x_{1}, T x_{1}\right), d\left(x_{2}, T x_{2}\right), d\left(x_{1}, T x_{2}\right), d\left(x_{2}, T x_{1}\right)\right\} .
\end{aligned}
$$

Since $T$ is a partial $q$-set-valued quasi-contraction and $\left(x_{1}, x_{2}\right) \in \bigwedge_{\alpha}$, we obtain

$$
H\left(T x_{1}, T x_{2}\right) \leq q \max \left\{d\left(x_{1}, x_{2}\right), d\left(x_{1}, T x_{1}\right), d\left(x_{2}, T x_{2}\right), d\left(x_{1}, T x_{2}\right), d\left(x_{2}, T x_{1}\right)\right\} .
$$


From (3.4) and (3.5), we have

$$
\begin{aligned}
d\left(x_{2}, x_{3}\right) & \leq(q+\varepsilon) \max \left\{d\left(x_{1}, x_{2}\right), d\left(x_{1}, T x_{1}\right), d\left(x_{2}, T x_{2}\right), d\left(x_{1}, T x_{2}\right), d\left(x_{2}, T x_{1}\right)\right\} \\
& =\beta \max \left\{d\left(x_{1}, x_{2}\right), d\left(x_{1}, T x_{1}\right), d\left(x_{2}, T x_{2}\right), d\left(x_{1}, T x_{2}\right), d\left(x_{2}, T x_{1}\right)\right\} .
\end{aligned}
$$

By induction, we can construct a sequence $\left\{x_{n}\right\}$ in $X$ such that, for each $n \in \mathbb{N}$, we have

$$
x_{n} \in T x_{n-1}, \quad\left(x_{n-1}, x_{n}\right) \in \bigwedge_{\alpha}
$$

and

$$
\begin{aligned}
& d\left(x_{n}, x_{n+1}\right) \\
& \quad \leq \beta \max \left\{d\left(x_{n-1}, x_{n}\right), d\left(x_{n-1}, T x_{n-1}\right), d\left(x_{n}, T x_{n}\right), d\left(x_{n-1}, T x_{n}\right), d\left(x_{n}, T x_{n-1}\right)\right\} .
\end{aligned}
$$

If there exists $\widehat{n} \in \mathbb{N}$ such that $x_{\widehat{n}-1}=x_{\widehat{n}}$, then $x_{\widehat{n}} \in T x_{\widehat{n}}$ and then the proof is complete. For the rest, we will assume that $x_{n-1} \neq x_{n}$, that is, $d\left(x_{n-1}, x_{n}\right)>0$, for all $n \in \mathbb{N}$. Now we obtain, for all $n \in \mathbb{N}$,

$$
\begin{aligned}
d\left(x_{n}, x_{n+1}\right) & \leq \beta \max \left\{d\left(x_{n-1}, x_{n}\right), d\left(x_{n-1}, T x_{n-1}\right), d\left(x_{n}, T x_{n}\right), d\left(x_{n-1}, T x_{n}\right), d\left(x_{n}, T x_{n-1}\right)\right\} \\
& \leq \beta \max \left\{d\left(x_{n-1}, x_{n}\right), d\left(x_{n-1}, x_{n}\right), d\left(x_{n}, x_{n+1}\right), d\left(x_{n-1}, x_{n+1}\right), d\left(x_{n}, x_{n}\right)\right\} \\
& \leq \beta \max \left\{d\left(x_{n-1}, x_{n}\right), d\left(x_{n}, x_{n+1}\right), s\left[d\left(x_{n-1}, x_{n}\right)+d\left(x_{n}, x_{n+1}\right)\right]\right\} \\
& \leq \beta s\left[d\left(x_{n-1}, x_{n}\right)+d\left(x_{n}, x_{n+1}\right)\right]
\end{aligned}
$$

and hence

$$
d\left(x_{n}, x_{n+1}\right) \leq \gamma d\left(x_{n-1}, x_{n}\right)
$$

where $\gamma:=\frac{\beta s}{1-\beta s}$.

Since $s \geq 1, \beta=\frac{1}{2}\left(\frac{1}{s^{2}+s}+q\right)$, and $q<\frac{1}{s^{2}+s}$, we get

$$
\gamma s<1
$$

From (3.7), (3.8), and Lemma 2.19, we see that $\left\{x_{n}\right\}$ is a Cauchy sequence in $X$. By the completeness of $X$, there exists $u \in X$ such that

$$
\lim _{n \rightarrow \infty} d\left(x_{n}, u\right)=0
$$

Next, we will prove that $d(u, T u)=0$. By the condition (iii), we have $\left(x_{n}, u\right) \in \bigwedge_{\alpha}$, for all $n \in \mathbb{N}$. From Lemma 2.16 and (3.1), for each $n \in \mathbb{N}$, we get

$$
\begin{aligned}
d(u, T u) & \leq s\left[d\left(u, x_{n+1}\right)+d\left(x_{n+1}, T u\right)\right] \\
& \leq s\left[d\left(u, x_{n+1}\right)+H\left(T x_{n}, T u\right)\right] \\
& \leq s\left[d\left(u, x_{n+1}\right)+q \max \left\{d\left(x_{n}, u\right), d\left(x_{n}, T x_{n}\right), d(u, T u), d\left(x_{n}, T u\right), d\left(u, T x_{n}\right)\right\}\right]
\end{aligned}
$$




$$
\begin{aligned}
\leq & s\left[d\left(u, x_{n+1}\right)+q \max \left\{d\left(x_{n}, u\right), d\left(x_{n}, x_{n+1}\right), d(u, T u), d\left(x_{n}, T u\right), d\left(u, x_{n+1}\right)\right\}\right] \\
\leq & s\left[d\left(u, x_{n+1}\right)+q \max \left\{d\left(x_{n}, u\right), s\left[d\left(x_{n}, u\right)+d\left(u, x_{n+1}\right)\right], d(u, T u),\right.\right. \\
& \left.\left.s\left[d\left(x_{n}, u\right)+d(u, T u)\right], d\left(u, x_{n+1}\right)\right\}\right] .
\end{aligned}
$$

Letting $n \rightarrow \infty$ in the above inequality, we have

$$
d(u, T u) \leq q s^{2} d(u, T u)
$$

It follows from $q<\frac{1}{s^{2}+s}$ that $q s^{2}<1$. From (3.10), we get $d(u, T u)=0$. Using Lemma 2.18, we have $u \in T u$, that is, $u$ is a fixed point of $T$. This completes the proof.

Theorem 3.3 Let $(X, d)$ be a complete $b$-metric space with coefficient $s \geq 1, \alpha: X \times$ $X \rightarrow[0, \infty)$ be a given mapping and $T: X \rightarrow P_{b, c l}(X)$ be a partial q-set-valued quasicontraction. Suppose that the following conditions hold:

(i) $T$ is $\alpha_{*}$-admissible;

(ii) there exist $x_{0} \in X$ and $x_{1} \in T x_{0}$ such that $\left(x_{0}, x_{1}\right) \in \bigwedge_{\alpha}$;

(iii) if $\left\{x_{n}\right\}$ is a sequence in $X$ such that $\left(x_{n}, x_{n+1}\right) \in \bigwedge_{\alpha}$, for all $n \in \mathbb{N}$, and $x_{n} \rightarrow x$ as $n \rightarrow \infty$, for some $x \in X$, then $\left(x_{n}, x\right) \in \bigwedge_{\alpha}$.

If we set $q<\frac{1}{s^{2}+s}$, then $T$ has a fixed point in $X$, that is, there exists $u \in X$ such that $u \in T u$.

Proof We can prove this result by using Theorem 3.2 and Remark 2.22.

Corollary 3.4 (Theorems 3.2, 3.3 in [10]) Let $(X, d)$ be a complete b-metric space with coefficient $s \geq 1, \alpha: X \times X \rightarrow[0, \infty)$ be a given mapping and $T: X \rightarrow P_{b, c l}(X)$ be a $q$-setvalued $\alpha$-quasi-contraction, that is, for all $x, y \in X$, we have

$$
\alpha(x, y) H(T x, T y) \leq q \max \{d(x, y), d(x, T x), d(y, T y), d(x, T y), d(y, T x)\}
$$

where $0 \leq q<1$. Suppose that the following conditions hold:

(i) $T$ is $\alpha$-admissible (or $\alpha_{*}$-admissible);

(ii) there exist $x_{0} \in X$ and $x_{1} \in T x_{0}$ such that $\left(x_{0}, x_{1}\right) \in \bigwedge_{\alpha}$;

(iii) if $\left\{x_{n}\right\}$ is a sequence in $X$ such that $\left(x_{n}, x_{n+1}\right) \in \bigwedge_{\alpha}$, for all $n \in \mathbb{N}$, and $x_{n} \rightarrow x$ as $n \rightarrow \infty$, for some $x \in X$, then $\left(x_{n}, x\right) \in \bigwedge_{\alpha}$.

If $q<\frac{1}{s^{2}+s}$, then $T$ has a fixed point in $X$, that is, there exists $u \in X$ such that $u \in T u$.

Proof We will show that a $q$-set-valued $\alpha$-quasi-contraction is a partial $q$-set-valued quasicontraction. Assume that $(x, y) \in \bigwedge_{\alpha}$ and so $\alpha(x, y) \geq 1$. Since $T$ is a $q$-set-valued $\alpha$-quasicontraction, we get

$$
\begin{aligned}
H(T x, T y) & \leq \alpha(x, y) H(T x, T y) \\
& \leq q \max \{d(x, y), d(x, T x), d(y, T y), d(x, T y), d(y, T x)\} .
\end{aligned}
$$

This implies that $T$ is a partial $q$-set-valued quasi-contraction. By Theorem 3.2 (or Theorem 3.3), we get the desired result. 
Corollary 3.5 Let $(X, d)$ be a complete $b$-metric space with coefficient $s \geq 1, \alpha: X \times X \rightarrow$ $[0, \infty)$ be a given mapping and let $T: X \rightarrow P_{b, c l}(X)$ satisfy

$$
(H(T x, T y)+\epsilon)^{\alpha(x, y)} \leq q \max \{d(x, y), d(x, T x), d(y, T y), d(x, T y), d(y, T x)\}+\epsilon,
$$

for all $x, y \in X$, where $0 \leq q<1$ and $\epsilon \geq 1$. Suppose that the following conditions hold:

(i) $T$ is $\alpha$-admissible (or $\alpha_{*}$-admissible);

(ii) there exist $x_{0} \in X$ and $x_{1} \in T x_{0}$ such that $\left(x_{0}, x_{1}\right) \in \bigwedge_{\alpha}$;

(iii) if $\left\{x_{n}\right\}$ is a sequence in $X$ such that $\left(x_{n}, x_{n+1}\right) \in \bigwedge_{\alpha}$, for all $n \in \mathbb{N}$, and $x_{n} \rightarrow x$ as $n \rightarrow \infty$, for some $x \in X$, then $\left(x_{n}, x\right) \in \bigwedge_{\alpha}$.

If $q<\frac{1}{s^{2}+s}$, then $T$ has a fixed point in $X$, that is, there exists $u \in X$ such that $u \in T u$.

Proof We will show that $T$ is a partial $q$-set-valued quasi-contraction. Suppose that $(x, y) \in$ $\bigwedge_{\alpha}$ and then $\alpha(x, y) \geq 1$. From (3.12), we get

$$
\begin{aligned}
H(T x, T y)+\epsilon & \leq(H(T x, T y)+\epsilon)^{\alpha(x, y)} \\
& \leq q \max \{d(x, y), d(x, T x), d(y, T y), d(x, T y), d(y, T x)\}+\epsilon,
\end{aligned}
$$

that is,

$$
H(T x, T y) \leq q \max \{d(x, y), d(x, T x), d(y, T y), d(x, T y), d(y, T x)\} .
$$

This implies that $T$ is a partial $q$-set-valued quasi-contraction. By Theorem 3.2 (or Theorem 3.3), we get the desired result.

Corollary 3.6 Let $(X, d)$ be a complete $b$-metric space with coefficient $s \geq 1, \alpha: X \times X \rightarrow$ $[0, \infty)$ be a given mapping and $T: X \rightarrow P_{b, c l}(X)$ satisfies

$$
(\alpha(x, y)-1+\epsilon)^{H(T x, T y)} \leq \epsilon^{q \max \{d(x, y), d(x, T x), d(y, T y), d(x, T y), d(y, T x)\}},
$$

for all $x, y \in X$, where $0 \leq q<1$ and $\epsilon>1$. Suppose that the following conditions hold:

(i) $T$ is $\alpha$-admissible (or $\alpha_{*}$-admissible);

(ii) there exist $x_{0} \in X$ and $x_{1} \in T x_{0}$ such that $\left(x_{0}, x_{1}\right) \in \bigwedge_{\alpha}$;

(iii) if $\left\{x_{n}\right\}$ is a sequence in $X$ such that $\left(x_{n}, x_{n+1}\right) \in \bigwedge_{\alpha}$, for all $n \in \mathbb{N}$, and $x_{n} \rightarrow x$ as $n \rightarrow \infty$, for some $x \in X$, then $\left(x_{n}, x\right) \in \bigwedge_{\alpha}$.

If $q<\frac{1}{s^{2}+s}$, then $T$ has a fixed point in $X$, that is, there exists $u \in X$ such that $u \in T u$.

Proof We will show that $T$ is a partial $q$-set-valued quasi-contraction. Suppose that $(x, y) \in$ $\bigwedge_{\alpha}$ and then $\alpha(x, y) \geq 1$. From (3.13), we get

$$
\begin{aligned}
\epsilon^{H(T x, T y)} & \leq(\alpha(x, y)-1+\epsilon)^{H(T x, T y)} \\
& \leq \epsilon^{q \max \{d(x, y), d(x, T x), d(y, T y), d(x, T y), d(y, T x)\}} .
\end{aligned}
$$

It follows from $\epsilon>1$ that

$$
H(T x, T y) \leq q \max \{d(x, y), d(x, T x), d(y, T y), d(x, T y), d(y, T x)\} .
$$


This implies that $T$ is a partial $q$-set-valued quasi-contraction. By Theorem 3.2 (or Theorem 3.3), we get the desired result.

Corollary 3.7 (Theorem 2.2 in [8]) Let $(X, d)$ be a complete b-metric space with coefficient $s \geq 1$ and $T: X \rightarrow P_{b, c l}(X)$ be a q-set-valued quasi-contraction. If $q<\frac{1}{s^{2}+s}$, then $T$ has a fixed point in $X$, that is, there exists $u \in X$ such that $u \in T u$.

Proof Set $\alpha(x, y)=1$, for all $x, y \in X$. By Theorem 3.2 (or Theorem 3.3), we obtain the desired result.

Remark 3.8 If we take $s=1$ (it corresponds to the case of metric spaces), then the condition of $q$ in Theorem 3.2 becomes $q<\frac{1}{2}$. Therefore, Theorems 3.2 and 3.3 are generalization of several known fixed point results in metric spaces. Also Theorem 3.2 is a generalization of Theorem 3.2 and 3.3 of Sintunavarat et al. [10], Theorem 2.2 of Aydi et al. [8], main results of Amini-Harandi [9], Daffer and Kaneko [26], Rouhani and Moradi [27], and Singh et al. [14].

The following example shows that Theorem 3.2 properly generalizes Theorem 2.2 of Aydi et al. [8].

Example 3.9 Let $X=\mathbb{R}$ and the functional $d: X \times X \rightarrow \mathbb{R}_{+}$defined by

$$
d(x, y):=|x-y|^{2},
$$

for all $x, y \in X$. Clearly, $(X, d)$ is a complete $b$-metric space with coefficient $s=2$. Define set-valued mapping $T: X \rightarrow P_{b, c l}(X)$ by

$$
T x= \begin{cases}{[x, \max \{x,-10\}],} & x \in(-\infty, 0), \\ {\left[0, \frac{x}{10}\right],} & x \in[0,2], \\ {[\min \{x, 7\}, x],} & x \in(2, \infty),\end{cases}
$$

and $\alpha: X \times X \rightarrow[0, \infty)$ by

$$
\alpha(x, y)= \begin{cases}3^{\ln (x+y+e)}, & x, y \in[0,2], \\ 0, & \text { otherwise. }\end{cases}
$$

We obtain

$$
H(T 0, T 6)=36
$$

and

$$
\max \{d(0,6), d(0, T 0), d(6, T 6), d(0, T 6), d(6, T 0)\}=36 .
$$

Therefore,

$$
H(T 0, T 6)>q \max \{d(0,6), d(0, T 0), d(6, T 6), d(0, T 6), d(6, T 0)\},
$$


for all $0 \leq q<1$. This implies that the contraction condition of Theorem 2.2. of Aydi et al. [8] is not true for this case. Therefore, Theorem 2.2 cannot be used to claim the existence of fixed point of $T$.

Next, we show that Theorem 3.2 can be applied for this case. First of all, we show that $T$ is a partial $q$-set-valued quasi-contraction mapping, where $q=\frac{1}{100}$. Assume that

$$
(x, y) \in \bigwedge_{\alpha}=\{(x, y) \in X \times X: \alpha(x, y) \geq 1\}=[0,2] \times[0,2] .
$$

Then we have

$$
\begin{aligned}
H(T x, T y) & =\left|\frac{x}{10}-\frac{y}{10}\right|^{2} \\
& =\frac{|x-y|^{2}}{100} \\
& =q d(x, y) \\
& \leq q \max \{d(x, y), d(x, T x), d(y, T y), d(x, T y), d(y, T x)\} .
\end{aligned}
$$

This shows that $T$ is a partial $q$-set-valued quasi-contraction mapping. Also we have

$$
q=\frac{1}{100}<\frac{1}{6}=\frac{1}{s^{2}+s} .
$$

It is easy to see that $T$ is an $\alpha$-admissible mapping. We find that there exist $x_{0}=2$ and $x_{1}=0.1 \in T x_{0}$ for which $\left(x_{0}, x_{1}\right) \in \bigwedge_{\alpha}$. Further, for any sequence $\left\{x_{n}\right\}$ in $X$ with $x_{n} \rightarrow x$ as $n \rightarrow \infty$, for some $x \in X$, and $\left(x_{n}, x_{n+1}\right) \in \bigwedge_{\alpha}$, for all $n \in \mathbb{N}$, we see that $\left(x_{n}, x\right) \in \bigwedge_{\alpha}$, for all $n \in \mathbb{N}$.

Therefore, all hypotheses of Theorem 3.2 are satisfied and so $T$ has a fixed point. In this case, $T$ have infinitely many fixed points.

\section{Consequences}

\subsection{Fixed point results of single-valued mappings}

In this section, we give the fixed point result for single-valued mappings. Before presenting our results, we introduce the new concept of a partial $q$-single-valued quasi-contraction mapping.

Definition 4.1 Let $(X, d)$ be a $b$-metric space and $\alpha: X \times X \rightarrow[0, \infty)$ be a mapping. The single-valued mapping $t: X \rightarrow X$ is said to be a partial $q$-single-valued quasi-contraction if

$$
(x, y) \in \bigwedge_{\alpha} \quad \Longrightarrow \quad d(t x, t y) \leq q \max \{d(x, y), d(x, t x), d(y, t y), d(x, t y), d(y, t x)\}
$$

where $0 \leq q<1$.

Next, we give the fixed point result for partial $q$-single-valued quasi-contraction mapping.

Theorem 4.2 Let $(X, d)$ be a complete b-metric space with coefficient $s \geq 1, \alpha: X \times X \rightarrow$ $[0, \infty)$ be a given mapping and $t: X \rightarrow X$ be a partial q-single-valued quasi-contraction. Suppose that the following conditions hold: 
(i) $t$ is $\alpha$-admissible;

(ii) there exists $x_{0} \in X$ such that $\left(x_{0}, t x_{0}\right) \in \bigwedge_{\alpha}$;

(iii) if $\left\{x_{n}\right\}$ is a sequence in $X$ such that $\left(x_{n}, x_{n+1}\right) \in \bigwedge_{\alpha}$, for all $n \in \mathbb{N}$, and $x_{n} \rightarrow x$ as $n \rightarrow \infty$, for some $x \in X$, then $\left(x_{n}, x\right) \in \bigwedge_{\alpha}$.

If $q<\frac{1}{s^{2}+s}$, then $t$ has a fixed point in $X$, that is, there exists $u \in X$ such that $u=t u$.

Proof It follows by applying Theorem 3.2 or Theorem 3.3.

Remark 4.3 Theorem 4.2 is an extension of Corollary 3.8 of Sintunavarat et al. [10], Corollary 2.4 of Aydi et al. [8], and the result of Ćirić [28].

Example 4.4 Let $X=\mathbb{R}$ and the functional $d: X \times X \rightarrow \mathbb{R}_{+}$defined by

$$
d(x, y):=|x-y|^{2}
$$

for all $x, y \in X$. Clearly, $(X, d)$ is a complete $b$-metric space with coefficient $s=2$. Define single-valued mapping $t: X \rightarrow X$ by

$$
t x= \begin{cases}\max \{x,-3\}, & x \in(-\infty, 0), \\ \frac{x}{3}, & x \in[0,1], \\ x^{3}, & x \in(1, \infty),\end{cases}
$$

and $\alpha: X \times X \rightarrow[0, \infty)$ by

$$
\alpha(x, y)= \begin{cases}2, & x, y \in[0,1] \\ 0.2, & \text { otherwise }\end{cases}
$$

We obtain

$$
d(t 0, t 2)=64
$$

and

$$
\max \{d(0,2), d(0, t 0), d(2, t 2), d(0, t 2), d(2, t 0)\}=64
$$

Therefore,

$$
d(t 0, t 2)>q \max \{d(0,2), d(0, t 0), d(2, t 2), d(0, t 2), d(2, t 0)\},
$$

for all $0 \leq q<1$. This implies that the contraction condition of Corollary 2.4 of Aydi et al. [8] is not true for this case. Therefore, Corollary 2.4 of Aydi et al. [8] cannot be used to claim the existence of fixed point of $t$.

Next, we show that Theorem 4.2 can be applying for this case. First of all, we show that $t$ is a partial $q$-single-valued quasi-contraction mapping, where $q=\frac{1}{9}$. Assume that $(x, y) \in \bigwedge_{\alpha}=\{(x, y) \in X \times X: \alpha(x, y) \geq 1\}=[0,1] \times[0,1]$. We obtain

$$
d(t x, t y)=\left|\frac{x}{3}-\frac{y}{3}\right|^{2}
$$




$$
\begin{aligned}
& =\frac{|x-y|^{2}}{9} \\
& =q d(x, y) \\
& \leq q \max \{d(x, y), d(x, t x), d(y, t y), d(x, t y), d(y, t x)\} .
\end{aligned}
$$

This shows that $t$ is a partial $q$-single-valued quasi-contraction mapping. Also we have

$$
q=\frac{1}{9}<\frac{1}{6}=\frac{1}{s^{2}+s} .
$$

It is easy to see that $t$ is an $\alpha$-admissible mapping.

We find that there exists $x_{0}=0.3$ such that $\left(x_{0}, t x_{0}\right)=(0.3,0.1) \in \bigwedge_{\alpha}$. Further, for any sequence $\left\{x_{n}\right\}$ in $X$ with $x_{n} \rightarrow x$ as $n \rightarrow \infty$, for some $x \in X$, and $\left(x_{n}, x_{n+1}\right) \in \bigwedge_{\alpha}$, for all $n \in \mathbb{N}$, we obtain $\left(x_{n}, x\right) \in \bigwedge_{\alpha}$, for all $n \in \mathbb{N}$, since $[0,1]$ is closed.

Therefore, all hypotheses of Theorem 4.2 are satisfied and so $t$ has a fixed point, that is, a point $0 \in X$.

\subsection{Fixed point results on $b$-metric space endowed with an arbitrary binary relation}

In this section, we give the fixed point results on a $b$-metric space endowed with an arbitrary binary relation. Before presenting our results, we give the following definitions.

Definition 4.5 Let $(X, d)$ be a $b$-metric space and $\mathcal{R}$ be a binary relation over $X$. We say that $T: X \rightarrow P_{b, c l}(X)$ is a weakly preserving mapping if for each $x \in X$ and $y \in T x$ with $x \mathcal{R} y$, we have $y \mathcal{R} z$, for all $z \in T y$.

Definition 4.6 Let $(X, d)$ be a $b$-metric space and $\mathcal{R}$ be a binary relation over $X$. The set-valued mapping $T: X \rightarrow P_{b, c l}(X)$ is said to be a $q$-set-valued quasi-contraction with respect to $\mathcal{R}$ if, for all $x, y \in X$, we have

$$
x \mathcal{R} y \quad \Longrightarrow \quad H(T x, T y) \leq q \max \{d(x, y), d(x, T x), d(y, T y), d(x, T y), d(y, T x)\}
$$

where $0 \leq q<1$.

Theorem 4.7 Let $(X, d)$ be a complete b-metric space with coefficient $s \geq 1, \mathcal{R}$ be a binary relation over $X$, and $T: X \rightarrow P_{b, c l}(X)$ be a q-set-valued quasi-contraction with respect to $\mathcal{R}$. Suppose that the following conditions hold:

(i) $T$ is a weakly preserving mapping;

(ii) there exist $x_{0} \in X$ and $x_{1} \in T x_{0}$ such that $x_{0} \mathcal{R} x_{1}$;

(iii) if $\left\{x_{n}\right\}$ is a sequence in $X$ such that $x_{n} \mathcal{R} x_{n+1}$, for all $n \in \mathbb{N}$, and $x_{n} \rightarrow x$ as $n \rightarrow \infty$, for some $x \in X$, then $x_{n} \mathcal{R} x$.

If $q<\frac{1}{s^{2}+s}$, then $T$ has a fixed point in $X$, that is, there exists $u \in X$ such that $u \in T u$.

Proof Consider the mapping $\alpha: X \times X \rightarrow[0, \infty)$ defined by

$$
\alpha(x, y)= \begin{cases}1 & \text { if } x \mathcal{R} y \\ 0 & \text { otherwise }\end{cases}
$$


From condition (ii), we get $\alpha\left(x_{0}, x_{1}\right) \geq 1$ and so $\left(x_{0}, x_{1}\right) \in \bigwedge_{\alpha}$. It follows from $T$ being a preserving mapping that $T$ is an $\alpha$-admissible mapping. Since $T$ is a $q$-set-valued quasicontraction with respect to $\mathcal{R}$, we have, for all $x, y \in X$,

$$
\begin{aligned}
& (x, y) \in \bigwedge_{\alpha} \\
& \quad \Longrightarrow \quad H(T x, T y) \leq q \max \{d(x, y), d(x, T x), d(y, T y), d(x, T y), d(y, T x)\} .
\end{aligned}
$$

This implies that $T$ is a partial $q$-set-valued quasi-contraction mapping. Now all the hypotheses of Theorem 3.2 are satisfied and so the existence of the fixed point of $T$ follows from Theorem 3.2.

Next, we give some special case of Theorem 4.7 in partially ordered $b$-metric spaces. Before we study the next results, we give the following definitions.

Definition 4.8 Let $X$ be a nonempty set. Then $(X, d, \preceq)$ is called a partially ordered $b$ metric space if $(X, d)$ is a $b$-metric space and $(X, \preceq)$ is a partially ordered space.

Definition 4.9 Let $(X, d, \preceq)$ be a partially ordered $b$-metric space. We say that $T: X \rightarrow$ $P_{b, c l}(X)$ is a weakly preserving mapping with $\preceq$ if for each $x \in X$ and $y \in T x$ with $x \preceq y$, we have $y \preceq z$, for all $z \in T y$.

Definition 4.10 Let $(X, d, \preceq)$ be a partially ordered $b$-metric space. The set-valued mapping $T: X \rightarrow P_{b, c l}(X)$ is said to be a $q$-set-valued quasi-contraction with respect to $\preceq$ if, for all $x, y \in X$, we have

$$
x \leq y \quad \Longrightarrow \quad H(T x, T y) \leq q \max \{d(x, y), d(x, T x), d(y, T y), d(x, T y), d(y, T x)\},
$$

where $0 \leq q<1$.

Corollary 4.11 Let $(X, d, \preceq)$ be a complete partially ordered b-metric space with coefficient $s \geq 1$ and $T: X \rightarrow P_{b, c l}(X)$ be a q-set-valued quasi-contraction with respect to $\preceq$. Suppose that the following conditions hold:

(i) $T$ is a weakly preserving mapping with $\preceq$;

(ii) there exist $x_{0} \in X$ and $x_{1} \in T x_{0}$ such that $x_{0} \leq x_{1}$;

(iii) if $\left\{x_{n}\right\}$ is a sequence in $X$ such that $x_{n} \preceq x_{n+1}$, for all $n \in \mathbb{N}$, and $x_{n} \rightarrow x$ as $n \rightarrow \infty$, for some $x \in X$, then $x_{n} \preceq x$.

If we set $q<\frac{1}{s^{2}+s}$, then $T$ has a fixed point in $X$, that is, there exists $u \in X$ such that $u \in T u$.

Proof The result follows from Theorem 4.7 by considering the binary relation $\preceq$.

\subsection{Fixed point results on $b$-metric spaces endowed with a graph}

Throughout this section, let $(X, d)$ be a $b$-metric space. A set $\{(x, x): x \in X\}$ is called a diagonal of the Cartesian product $X \times X$ and is denoted by $\Delta$. Consider a directed graph $G$ such that the set $V(G)$ of its vertices coincides with $X$ and the set $E(G)$ of its edges contains all loops, i.e., $\Delta \subseteq E(G)$. We assume that $G$ has no parallel edges, so we can identify $G$ with the pair $(V(G), E(G))$. Moreover, we may treat $G$ as a weighted graph by assigning to each edge the distance between its vertices. 
In this section, we give the fixed point results for set-valued mappings in a $b$-metric space endowed with a graph. Before presenting our results, we will introduce new definitions in a $b$-metric space endowed with a graph.

Definition 4.12 Let $(X, d)$ be a $b$-metric space endowed with a graph $G$ and $T: X \rightarrow$ $P_{b, c l}(X)$ be set-valued mapping. We say that $T$ weakly preserves the edges of $G$ if for each $x \in X$ and $y \in T x$ with $(x, y) \in E(G)$ implies $(y, z) \in E(G)$, for all $z \in T y$.

Definition 4.13 Let $(X, d)$ be a $b$-metric space endowed with a graph $G$. A set-valued mapping $T: X \rightarrow P_{b, c l}(X)$ is said to be a $q$ - $G$-set-valued quasi-contraction if, for all $x, y \in X$, we have

$$
\begin{aligned}
& (x, y) \in E(G) \\
& \quad \Longrightarrow \quad H(T x, T y) \leq q \max \{d(x, y), d(x, T x), d(y, T y), d(x, T y), d(y, T x)\}
\end{aligned}
$$

where $0 \leq q<1$.

Example 4.14 Let $X$ be a nonempty set. Any mapping $T: X \rightarrow P_{b, c l}(X)$ defined by $T x=$ $\{a\}$, where $a \in X$, is a $q$-G-set-valued quasi-contraction for any graph $G$ with $V(G)=X$.

Example 4.15 Let $X$ be a nonempty set. Any mapping $T: X \rightarrow P_{b, c l}(X)$ is trivially a $q$-Gset-valued quasi-contraction, where $G=(V(G), E(G))=(X, \Delta)$.

Definition 4.16 Let $(X, d)$ be a $b$-metric space endowed with a graph $G$. We say that $X$ has $G$-regular property if given $x \in X$ and sequence $\left\{x_{n}\right\}$ in $X$ such that $x_{n} \rightarrow x$ as $n \rightarrow \infty$ and $\left(x_{n}, x_{n+1}\right) \in E(G)$, for all $n \in \mathbb{N}$, then $\left(x_{n}, u\right) \in E(G)$, for all $n \in \mathbb{N}$.

Here, we give a fixed point result for set-valued mappings in a $b$-metric space endowed with a graph.

Theorem 4.17 Let $(X, d)$ be a complete $b$-metric space with coefficient $s \geq 1$ and endowed with a graph $G$ and let $T: X \rightarrow P_{b, c l}(X)$ be a $q$-G-set-valued quasi-contraction. Suppose that the following conditions hold:

(i) $T$ weakly preserves edges of $G$;

(ii) there exist $x_{0} \in X$ and $x_{1} \in T x_{0}$ such that $\left(x_{0}, x_{1}\right) \in E(G)$;

(iii) $X$ has $G$-regular property.

If $q<\frac{1}{s^{2}+s}$, then $T$ has a fixed point in $X$, that is, there exists $u \in X$ such that $u \in T u$.

Proof Consider the mapping $\alpha: X \times X \rightarrow[0, \infty)$ defined by

$$
\alpha(x, y)= \begin{cases}1, & (x, y) \in E(G) \\ 0, & \text { otherwise }\end{cases}
$$

Since $T$ is a $q$-G-set-valued quasi-contraction, we have, for all $x, y \in X$,

$$
\begin{aligned}
& (x, y) \in \bigwedge_{\alpha} \\
& \quad \Longrightarrow \quad H(T x, T y) \leq q \max \{d(x, y), d(x, T x), d(y, T y), d(x, T y), d(y, T x)\} .
\end{aligned}
$$


This implies that $T$ is a partial $q$-set-valued quasi-contraction.

By construction of $\alpha$ and condition (i), we find that $T$ is $\alpha$-admissible. From condition (ii) and the construction of $\alpha$, we get $\alpha\left(x_{0}, x_{1}\right) \geq 1$ and thus $\left(x_{0}, x_{1}\right) \in \bigwedge_{\alpha}$. Using G-regular property of $X$, the result is obtained that the condition (iii) in Theorem 3.2 holds. Now all the hypotheses of Theorem 3.2 are satisfied and so the existence of the fixed point of $T$ follows from Theorem 3.2.

\section{Existence of a solution for an integral equation}

In this section, we prove the existence theorem for a solution of the following integral equation by using Theorem 4.2:

$$
x(c)=\phi(c)+\int_{a}^{b} K(c, r, x(r)) d r
$$

where $b>a \geq 0, x \in C[a, b]$ (the set of continuous real functions defined on $[a, b] \subseteq \mathbb{R}$ ), $\phi:[a, b] \rightarrow \mathbb{R}$, and $K:[a, b] \times[a, b] \times \mathbb{R} \rightarrow \mathbb{R}$ are given mappings.

Theorem 5.1 Suppose that the following hypotheses hold:

( $\left.\mathrm{I}_{1}\right) K:[a, b] \times[a, b] \times \mathbb{R} \rightarrow \mathbb{R}$ is continuous;

$\left(\mathrm{I}_{2}\right)$ there exists $p \geq 1$ satisfies the following condition for each $r, c \in[a, b]$ and $x, y \in X$ with $x(w) \leq y(w)$, for all $w \in[a, b]:$

$$
|K(c, r, x(r))-K(c, r, y(r))| \leq \xi(c, r)|x(r)-y(r)|,
$$

where $\xi:[a, b] \times[a, b] \rightarrow[0, \infty)$ is a continuous function satisfying

$$
\sup _{c \in[a, b]}\left(\int_{a}^{b} \xi(c, r)^{p} d r\right) \leq \frac{1}{\left(2^{p-1}+1\right)(b-a)^{p-1}}
$$

$\left(\mathrm{I}_{3}\right)$ there exists $x_{0} \in X$ such that $x_{0}(c) \leq\left(t x_{0}\right)(c)$, for all $c \in[a, b]$.

Then the integral equation (5.1) has a solution $x \in X$.

Proof Let $X=C[a, b]$ and let $t: X \rightarrow X$ be a mapping defined by

$$
(t x)(c)=\int_{a}^{b} K(c, r, x(r)) d r
$$

for all $x \in X$ and $c \in[a, b]$. Clearly, $X$ with the $b$-metric $d: X \times X \rightarrow \mathbb{R}_{+}$given by

$$
d(x, y)=\sup _{c \in[a, b]}|x(c)-y(c)|^{p}
$$

for all $x, y \in X$, is a complete $b$-metric space with coefficient $s=2^{p-1}$.

Define a mapping $\alpha: X \times X \rightarrow[0, \infty)$ by

$$
\alpha(x, y)= \begin{cases}1, & x(c) \leq y(c), \text { for all } c \in[a, b] \\ 0, & \text { otherwise }\end{cases}
$$


It is easy to see that $t$ is an $\alpha$-admissible mapping. From $\left(\mathrm{I}_{3}\right)$, we have $\left(x_{0}, t x_{0}\right) \in \bigwedge_{\alpha}$. Also we find that condition (iii) in Theorem 4.2 holds (see [29]).

Next, we show that $t$ is a partial $q$-single-valued quasi-contraction mapping with $q=$ $\frac{1}{2^{p-1}+1}<\frac{1}{2^{p-1}\left(2^{p-1}+1\right)}=\frac{1}{s^{2}+s}$. Let $1 \leq p^{\prime}<\infty$ with $\frac{1}{p}+\frac{1}{p^{\prime}}=1$. Now, let $x, y \in X$ be such that $(x, y) \in \bigwedge_{\alpha}$, that is, $x(c) \leq y(c)$, for all $c \in[a, b]$. From $\left(\mathrm{I}_{1}\right),\left(\mathrm{I}_{2}\right)$, and the Hölder inequality, for each $s \in[a, b]$ we have

$$
\begin{aligned}
|(t x)(s)-(t y)(s)|^{p} & \leq\left(\int_{a}^{b}|K(c, r, x(r))-K(c, r, y(r))| d r\right)^{p} \\
& \leq\left[\left(\int_{a}^{b} 1^{q} d r\right)^{\frac{1}{p^{p}}}\left(\int_{a}^{b}|K(c, r, x(r))-K(c, r, y(r))|^{p} d r\right)^{\frac{1}{p}}\right]^{p} \\
& \leq(b-a)^{\frac{p}{p}}\left(\int_{a}^{b}(\xi(c, r))^{p}|x(r)-y(r)|^{p} d r\right) \\
& =(b-a)^{\frac{p}{p^{\prime}}}\left(\int_{a}^{b} \xi(c, r)^{p} d(x, y) d r\right) \\
& \leq(b-a)^{\frac{p}{p^{\prime}}}\left(\int_{a}^{b} \xi(c, r)^{p} d(x, y) d r\right) \\
& =(b-a)^{p-1}\left(\int_{a}^{b} \xi(c, r)^{p} d r\right)(d(x, y)) \\
& \leq \frac{1}{2^{p-1}+1} \max \{d(x, y), d(x, t x), d(y, t y), d(x, t y), d(y, t x)\} \\
& =q \max \{d(x, y), d(x, t x), d(y, t y), d(x, t y), d(y, t x)\} .
\end{aligned}
$$

This shows that

$$
d(t x, t y) \leq q \max \{d(x, y), d(x, t x), d(y, t y), d(x, t y), d(y, t x)\} .
$$

Therefore, by using Theorem 4.2, we see that $t$ has a fixed point, that is, there exists $x \in$ $X$ such that $x$ is a fixed point of $t$. This implies that $x$ is a solution for (5.1) because the existence of a solution of (5.1) is equivalent to the existence of a fixed point of $t$. This completes the proof.

\footnotetext{
Competing interests

The authors declare that they have no competing interests.
}

Authors' contributions

All authors contributed equally and significantly in writing this paper. All authors read and approved the final manuscript.

\section{Author details}

'Department of Mathematics, Faculty of Science, King Mongkut's University of Technology Thonburi (KMUTT), Bangkok, 10140, Thailand. ${ }^{2}$ Department of Mathematics and Statistics, Faculty of Science and Technology, Thammasat University Rangsit Center, Pathumthani, 12121, Thailand.

\section{Acknowledgements}

The second author would like to thank the Thailand Research Fund and Thammasat University under Grant No.

TRG5780013 for financial support during the preparation of this manuscript. 


\section{References}

1. Czerwik, S: Contraction mappings in b-metric spaces. Acta Math. Inform. Univ. Ostrav. 1, 5-11 (1993)

2. Abusalim, SM, Noorani, MSM: Fixed point and common fixed point theorems on ordered cone $b$-metric spaces. Abstr. Appl. Anal. 2013, Article ID 815289 (2013)

3. Boriceanu, M: Fixed point theory for multivalued generalized contraction on a set with two $b$-metrics. Stud. Univ. Babes-Bolyai, Math. 54(3), 3-14 (2009)

4. Boriceanu, M, Bota, M, Petrusel, A: Multivalued fractals in b-metric spaces. Cent. Eur. J. Math. 8(2), 367-377 (2010)

5. Czerwik, S, Dlutek, K, Singh, SL: Round-off stability of iteration procedures for operators in $b$-metric spaces. J. Natur. Phys. Sci. 11, 87-94 (1997)

6. Czerwik, S: Nonlinear set-valued contraction mappings in b-metric spaces. Atti Semin. Mat. Fis. Univ. Modena 46(2), 263-276 (1998)

7. Parvaneh, V, Roshan, JR, Radenovic, S: Existence of tripled coincidence point in ordered $b$-metric spaces and application to a system of integral equations. Fixed Point Theory Appl. 2013, Article ID 130 (2013)

8. Aydi, H, Bota, M-F, Karapınar, E, Mitrović, S: A fixed point theorem for set-valued quasicontractions in $b$-metric spaces. Fixed Point Theory Appl. 2012, Article ID 88 (2012)

9. Amini-Harandi, A: Fixed point theory for set-valued quasi-contraction maps in metric spaces. Appl. Math. Lett. 24(11), 1791-1794 (2011)

10. Sintunavarat, W, Plubtieng, S, Katchang, P: Fixed point result and applications on b-metric space endowed with an arbitrary binary relation. Fixed Point Theory Appl. 2013, Article ID 296 (2013)

11. Berinde, V: Generalized contractions in quasimetric spaces. In: Seminar on Fixed Point Theory, pp. 3-9 (1993)

12. Heinonen, J: Lectures on Analysis on Metric Spaces. Springer, Berlin (2001)

13. Roshan, JR, Parvaneh, V, Sedghi, S, Shobkolaei, N, Shatanawi, W: Common fixed points of almost generalized $(\psi, \varphi)_{s}$-contractive mappings in ordered $b$-metric spaces. Fixed Point Theory Appl. 2013, Article ID 159 (2013)

14. Singh, SL, Czerwik, S, Krol, K, Singh, A: Coincidences and fixed points of hybrid contractions. Tamsui Oxford Univ. J. Math. Sci. 24(4), 401-416 (2008)

15. Samet, B, Vetro, C, Vetro, P: Fixed point theorems for $\alpha$ - $\psi$-contractive type mappings. Nonlinear Anal. $75,2154-2165$ (2012)

16. Asl, JH, Rezapour, S, Shahzad, N: On fixed points of $\alpha-\psi$-contractive multifunctions. Fixed Point Theory Appl. 2012, Article ID 212 (2012)

17. Mohammadi, B, Rezapour, S, Shahzad, N: Some results on fixed points of $\alpha$ - $\psi$-Ćirić generalized multifunctions. Fixed Point Theory Appl. 2013, Article ID 24 (2013)

18. Agarwal, RP, Sintunavarat, W, Kumam, P: PPF dependent fixed point theorems for an $\alpha_{c}$-admissible non-self mapping in the Razumikhin class. Fixed Point Theory Appl. 2013, Article ID 280 (2013)

19. Fathollahi, S, Salimi, P, Sintunavarat, W, Vetro, P: On fixed points of $\alpha-\eta-\psi$-contractive multifunctions. Wulfenia $21(2)$, 353-365 (2014)

20. Hussain, N, Salimi, P, Latif, A: Fixed point results for single and set-valued $\alpha-\eta$ - $\psi$-contractive mappings. Fixed Point Theory Appl. 2013, Article ID 212 (2013)

21. Kutbi, MA, Sintunavarat, W: The existence of fixed point theorems via $w$-distance and $\alpha$-admissible mappings and applications. Abstr. Appl. Anal. 2013, Article ID 165434 (2013)

22. Latif, $\mathrm{A}$, Mongkolkeha, $\mathrm{C}$, Sintunavarat, W: Fixed point theorems for generalized $\alpha$ - $\beta$-weakly contraction mappings in metric spaces and applications. Sci. World 2014, Article ID 784207 (2014)

23. Latif, A, Gordji, ME, Karapınar, E, Sintunavarat, W: Fixed point results for generalized $(\alpha-\psi)$-Meir Keeler contractive mappings and applications. J. Inequal. Appl. 2014, Article ID 68 (2014)

24. Salimi, P, Latif, A, Hussain, N: Modified $\alpha$ - $\psi$-contractive mappings with applications. Fixed Point Theory Appl. 2013, Article ID 151 (2013)

25. Usman Ali, M, Kamran, T, Sintunavarat, W, Katchang, P: Mizoguchi-Takahashi's fixed point theorem with $\alpha, \eta$ functions. Abstr. Appl. Anal. 2013, Article ID 418798 (2013)

26. Daffer, PZ, Kaneko, H: Fixed points of generalized contractive multi-valued mappings. J. Math. Anal. Appl. 192, 655-666 (1995)

27. Rouhani, BD, Moradi, S: Common fixed point of multivalued generalized $(\psi, \phi)$-weak contractive mappings. Fixed Point Theory Appl. 2010, Article ID 708984 (2010)

28. Ćirić, LB: A generalization of Banach's contraction principle. Proc. Am. Math. Soc. 45, 267-273 (1974)

29. Nieto, JJ, Rodíguez-López, R: Existence and uniqueness of fixed points in partially ordered sets and applications to ordinary differential equations. Acta Math. Sin. Engl. Ser. 23, 2205-2212 (2007)

10.1186/1687-1812-2014-226

Cite this article as: Kumam and Sintunavarat: The existence of fixed point theorems for partial $q$-set-valued quasi-contractions in b-metric spaces and related results. Fixed Point Theory and Applications 2014, 2014:226 